\title{
Fabrication of bismuth nanowires with a silver nanocrystal shadowmask
}

\author{
S. H. Choi ${ }^{\mathrm{a}}$ and K. L. Wang \\ Electrical Engineering Department, University of California at Los Angeles, Los Angeles, \\ California 9095-1594
}

M. S. Leung, G. W. Stupian, N. Presser, B. A. Morgan, R. E. Robertson, M. Abraham, E. E. King, and M. B. Tueling

Electronics Technology Center, The Aerospace Corporation, El Segundo, California 90245-4691

S. W. Chung and J. R. Heath

Department of Chemistry and Biochemistry, University of California at Los Angeles, Los Angeles, California 90095-1569

\author{
S. L. Cho and J. B. Ketterson \\ Department of Physics and Astronomy, Northwestern University, Evanston, Illinois 60208
}

(Received 1 October 1999; accepted 3 January 2000)

\begin{abstract}
We fabricated bismuth $(\mathrm{Bi})$ nanowires with low energy electron beam lithography using silver $(\mathrm{Ag})$ nanocrystal shadowmasks and a subsequent chlorine reactive ion etching. Submicron-size metal contacts on the single Bi nanowire were successfully prepared by in situ focused ion beam metal deposition for transport measurements. The temperature dependent resistance measurements on the $50 \mathrm{~nm}$ wide Bi nanowires showed that the resistance increased with decreasing temperature, which is characteristic of semiconductors and insulators. (c) 2000 American Vacuum Society.
\end{abstract}

[S0734-2101(00)03704-0]

\section{INTRODUCTION}

Nanometer-size structures such as wires and dots have aroused considerable interests as ideal systems for testing predictions about quantum confinement and reduced dimensionality, and as building blocks for nanostructured materials. A more practical reason for the study of nanostructures is the ever present drive towards smaller sizes in the electronics industry. ${ }^{1}$ In particular, semimetallic bismuth (Bi) with very small effective mass and high carrier mobilities is reported to be a good candidate to study quantum-confinement effects in one-dimensional systems and a very promising material for thermoelectric applications. ${ }^{2}$

Recently, a number of fabrication techniques have been utilized to produce Bi nanowires. For example, filling porous anodic alumina with $\mathrm{Bi}$ from the liquid phase resulting in single-crystal nanowire arrays having the same crystal structure and lattice parameters as bulk. ${ }^{3}$ However, it would be difficult to make contacts to a single Bi wire to study its one-dimensional properties.

We previously demonstrated a new fabrication technique for the fabrication of nanometer-size polymethyl methacrylate (PMMA) patterns using Ag nanocrystal shadowmasks. ${ }^{4}$ In this work, we used same technique and a subsequent reactive ion etching to fabricate $\mathrm{Bi}$ nanowires. $100 \mathrm{~nm}$ wide platinum ( $\mathrm{Pt}$ ) lines were written to contact a $\mathrm{Bi}$ single nanowire by the FIB to permit transport measurement.

\section{EXPERIMENTAL RESULTS}

First, we prepared $40 \mathrm{~nm}$ thick Bi single-crystal films by molecular beam epitaxy (MBE) system. The substrates for

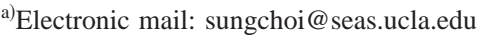

the MBE growth were indium doped semi-insulating CdTe(111)B pieces, $1 \mathrm{~cm}^{2}$ in size. X-ray diffraction showed only sharp $(000 l)$ peaks, which implied $c$-axis growth of $\mathrm{Bi}$ perpendicular to the substrates as shown in Fig. 1. Selfassembled high-aspect ratio $\mathrm{Ag}$ wire structures were transferred as a Langmuir-Schaeffer film to $40 \mathrm{~nm}$ thick $1 \%$ PMMA coated MBE-grown Bi/CdTe substrates. Substrates with the transferred $\mathrm{Ag}$ wires were exposed by a JEOL $6401 \mathrm{~F}$ field emission scanning electron microscope (FESEM) at $700 \mathrm{~V}$ to provide samples with an electron dose of $50 \mu \mathrm{C} / \mathrm{cm}^{2}$. At $700 \mathrm{~V}, 40 \mathrm{~nm}$ thick PMMA was thought to be exposed all the way to surface of Bi film. The penetration depth of electron in silver was found to be $4 \mathrm{~nm}$ at $700 \mathrm{~V}$ by a previous Monte Carlo simulation. ${ }^{4}$ That penetration depth is smaller than the thickness of Ag nanocrystal shadowmask. Following the electron beam exposure, a sample was developed for $1 \mathrm{~min}$ in a mixture of methyl isobutyl ketone and isopropanol in the ratio 1:3. A subsequent anisotropic reactive ion etching (RIE) process was carried out by a PlasmaMaster Model PME 1200 chlorine etcher. With a $\mathrm{BCl}_{3}$ to $\mathrm{Ar}_{2}$ mixture at $20 \mathrm{mTorr}$, and a plasma sustaining power of $200 \mathrm{~W}$, RIE process transferred Ag nanowire patterns to Bi/ CdTe substrates. The mask material is believed to consist of $30 \mathrm{~nm}$ thick $\mathrm{Ag}$ and $40 \mathrm{~nm}$ thick PMMA layers.

Its electronic orbital configuration of $\mathrm{Bi}$ implies that $\mathrm{Bi}$ prefers to have two ionization states. The following simple model of the $\mathrm{Bi}$ etching mechanism for the case of $\mathrm{Bi}^{+3}$ ionization state is proposed:

$$
\begin{aligned}
& \mathrm{Bi} \rightarrow \mathrm{Bi}^{+3}+3 e^{-} \\
& \frac{3}{2} \mathrm{Cl}_{2}+3 e^{-} \rightarrow 3 \mathrm{Cl}^{-}
\end{aligned}
$$




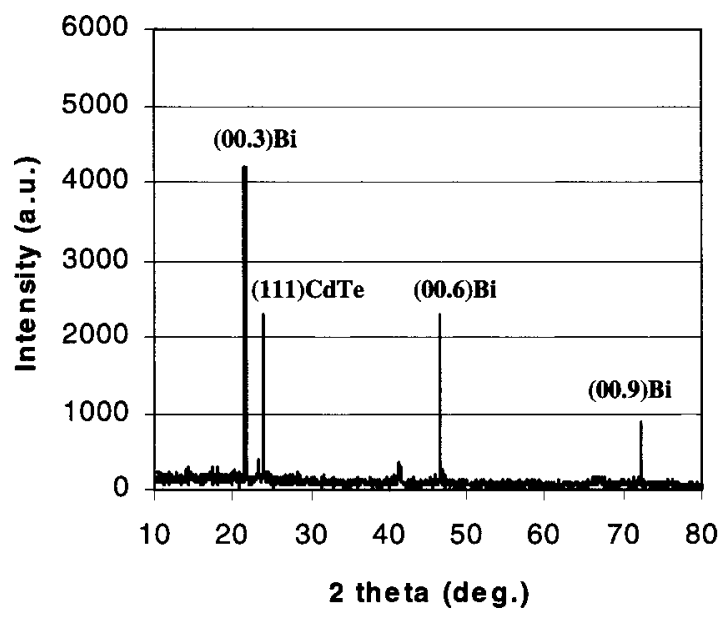

FIG. 1. X-ray diffraction pattern of MBE-grown Bi film on CdTe (111)B substrate.

$$
\mathrm{Bi}+\frac{3}{2} \mathrm{Cl}_{2} \rightarrow \mathrm{Bi}^{+3}+3 \mathrm{Cl}^{-} \rightarrow 3 \mathrm{BiCl}_{3}(\text { volatile }) .
$$

Following formation of $\mathrm{Bi}^{+3}$ and $\mathrm{Cl}^{-3}$ ions by plasma, volatile products of $\mathrm{BCl}_{3}$ were formed and washed away. $\mathrm{Ar}$ in the etch gas mixture might contribute to the reduction of the undercut profile. We have found a mixture of $\mathrm{BCl}_{3}$ and $\mathrm{Ar}_{2}$ to be an excellent choice of gases to be used in RIE of $\mathrm{Bi}$, producing vertical profiles and etching rates at about 100 $\mathrm{nm} / \mathrm{min}$. The RIE chemical mechanism for $\mathrm{Bi}$ is found to be similar to that of GaAs etching by chlorine gases. Through the RIE process, $50 \mathrm{~nm}$ wide and $40 \mathrm{~nm}$ high Bi nanowires were fabricated on CdTe substrates.

Submicron-size platinum $(\mathrm{Pt})$ contacts on a $\mathrm{Bi}$ single nanowire were prepared by in situ FIB metal deposition at 25 $\mathrm{kV}, 6 \mathrm{pA}$ to allow temperature dependent resistance measurements. Figure 2 shows the FESEM micrograph of a 50 $\mathrm{nm}$ wide, $40 \mathrm{~nm}$ high $\mathrm{Bi}$ nanowire to which Pt contacts were made by the FIB. The electrical resistance of the wire measured was typically on the order of $1-20 \Omega$. The temperature dependence of the resistance of $50 \mathrm{~nm}$ wide $\mathrm{Bi}$ nanowire is

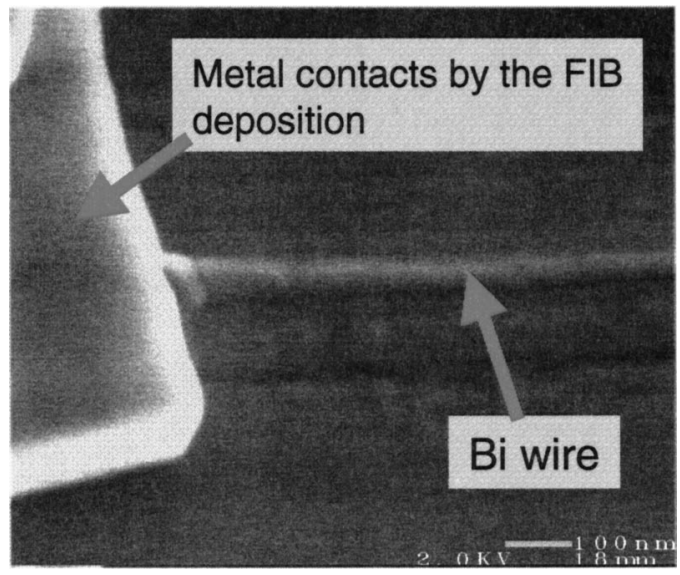

FIG. 2. FESEM micrograph of a $50 \mathrm{~nm}$ wide Bi nanowire with metal contacts. Pt contacts to the wire were prepared by in situ FIB metal deposition.

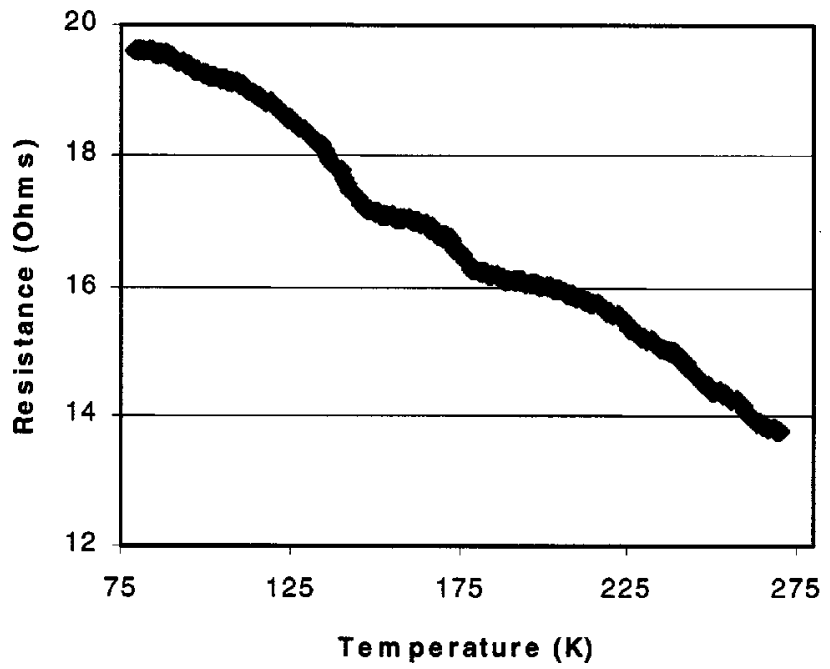

FIG. 3. Temperature dependence of the resistance of the $50 \mathrm{~nm}$ wide $\mathrm{Bi}$ nanowire.

shown in Fig. 3. The data show that resistance increases with decreasing temperature, which is characteristic of semiconductors and insulators. As quantum confinement is introduced into the $\mathrm{Bi}$ nanowire system, the external conduction subband and valence subband edges move in opposite directions to eventually form a positive energy band gap $\left(E_{g}\right)$ between the lowest $L$-point conduction subband edge and the highest $T$-point valence band edge, thereby leading to a semimetal-semiconductor transition $\left(E_{g}=0\right)$ as the wire size is decreased below the critical wire width of $\mathrm{Bi}$. Dresselhaus et al. found that $\mathrm{Bi}$ makes a transition at critical wire radius of $52 \mathrm{~nm} .^{5}$ In the Bi nanowires, the carrier mobility is suppressed by carrier confinement along the direction of wire and by surface imperfection.

\section{CONCLUSIONS}

In conclusion, $50 \mathrm{~nm}$ wide $\mathrm{Bi}$ nanowires were fabricated by low energy electron beam lithography using Ag nanocrystals as a shadowmask and a subsequent chlorine reactive ion etching process. Temperature dependent resistance measurements show that the Bi nanowire fabricated has semiconductor properties rather than metallic properties. Our method may be a good candidate of fabrication technique for studying $\mathrm{Bi}$ nanowires.

\section{ACKNOWLEDGMENTS}

The authors gratefully acknowledge support by the Augmentation Awards for Science and Engineering Research Training (AASERT) Fellowship Grant No. N00014-96-11258, the Office of Naval Research MURI, and ARO MURI and the U.S. Air Force under Contract No. F04701-93-C0094. One of the authors (J.R.H.) acknowledges the Office of Naval Research Contract No. N00014-981-0422, and the Packard Foundation. 
${ }^{1}$ A. P. Alivisatos, Science 271, 933 (1996).

${ }^{2}$ X. Sun, Z. Zhang, and M. S. Dresselhaus, Appl. Phys. Lett. 20, 4005 (1999).

${ }^{3}$ K. Liu, C. L. Chen, P. C. Searson, and K. Zhang, Appl. Phys. Lett. 73, 1436 (1998).
${ }^{4}$ S. H. Choi, K. L. Wang, M. S. Leung, G. W. Stupian, N. Presser, S. W. Chung, G. Markovich, S. H. Kim, and J. R. Heath, J. Vac. Sci. Technol. A 17, 1425 (1999).

${ }^{5}$ Z. Zhang, X. Sun, M. S. Dresselhaus, J. Ying, and J. P. Hermans, Appl. Phys. Lett. 73, 1589 (1998). 